\title{
Mycobacterium Survival Strategy Translated to Develop a Lipo-Peptide Based Fusion Inhibitor
}

\author{
Avijit Sardar, Aritraa Lahiri, Amirul Islam Mallick, Pradip Kumar Tarafdar
}

Submitted date: 05/05/2020 - Posted date: 07/05/2020

Licence: CC BY-NC-ND 4.0

Citation information: Sardar, Avijit; Lahiri, Aritraa; Mallick, Amirul Islam; Tarafdar, Pradip Kumar (2020): Mycobacterium Survival Strategy Translated to Develop a Lipo-Peptide Based Fusion Inhibitor. ChemRxiv. Preprint. https://doi.org/10.26434/chemrxiv.12249737.v1

The entry of enveloped viruses requires fusion of viral and host cell membranes. An effective fusion inhibitor aiming at impeding such virus-host cell membrane fusion may emerge as a broad-spectrum antiviral agent to neutralize the infection from an increasing diversity of harmful new viruses. Mycobacterium survives inside the phagosome of the host cells by inhibiting phagosome-lysosome fusion with the help of a coat protein coronin 1. Structural analysis of coronin 1 and other WD40-repeat containing protein suggest that the tryptophan-aspartic acid (WD) sequence is placed at distorted $\beta$-meander motif (more exposed) whereas the WD resides in regular $\beta$-meander motif in other WD40 proteins. The unique structural feature of coronin 1 was explored to identify a simple lipo-peptide sequence (lipid-WD), which effectively inhibit the membrane fusion by increasing interfacial order and decreasing water penetration, surface potential. The effective fusion inhibitory role of mycobacterium inspired lipo-dipeptide was applied to combat type 1 influenza virus (H1N1) infection as a 'broad spectrum' antiviral agent.

File list (1) 


\title{
Mycobacterium survival strategy translated to develop a lipo-peptide based fusion inhibitor
}

\author{
Avijit Sardar ${ }^{\mathrm{a}}$, Aritraa Lahiri ${ }^{\mathrm{b}}$, Amirul I. Mallick*b and Pradip K. Tarafdar*a \\ ${ }^{a}$ Department of Chemical Sciences, ${ }^{b}$ Department of Biological Sciences, Indian Institute of \\ Science Education and Research Kolkata, Mohanpur-741246, India
}

*Corresponding author:

Dr. Pradip Kumar Tarafdar

Department of Chemical Sciences

Indian Institute of Science Education and Research Kolkata

Mohanpur-741 246, India

E-mail: tarafdar@iiserkol.ac.in,srpradip@gmail.com

Dr. Amirul Islam Mallick

Department of Biological Sciences

Indian Institute of Science Education and Research Kolkata

Mohanpur-741 246, India

E-mail: amallick@iiserkol.ac.in

Keywords: Membrane fusion, enveloped virus, lipo-peptide inhibitor, water penetration, broad-spectrum antivirals. 


\begin{abstract}
The entry of enveloped viruses requires fusion of viral and host cell membranes. An effective fusion inhibitor aiming at impeding such virus-host cell membrane fusion may emerge as a broad-spectrum antiviral agent to neutralize the infection from an increasing diversity of harmful new viruses. Mycobacterium survives inside the phagosome of the host cells by inhibiting phagosome-lysosome fusion with the help of a coat protein coronin 1. Structural analysis of coronin 1 and other WD40-repeat containing protein suggest that the tryptophanaspartic acid (WD) sequence is placed at distorted $\beta$-meander motif (more exposed) whereas the WD resides in regular $\beta$-meander motif in other WD40 proteins. The unique structural feature of coronin 1 was explored to identify a simple lipo-peptide sequence (lipid-WD), which effectively inhibit the membrane fusion by increasing interfacial order and decreasing water penetration, surface potential. The effective fusion inhibitory role of mycobacterium inspired lipo-dipeptide was applied to combat type 1 influenza virus (H1N1) infection as a ‘broad spectrum’ antiviral agent.
\end{abstract}

\title{
Introduction
}

Emerging infectious diseases remain a major risk to public health and have serious impact on global economy. A large number of these diseases are zoonotic in nature and incidence of such zoonoses increases when human and environmental factors are compromised due to unintended overlap of previously distinct ecological domains. In one such recent protrude, a novel coronavirus (Covid-19) strain has emerged, infecting 3.6 million people globally and The World Health Organization declared the outbreak a pandemic. The disease count is fast escalating with no vaccine currently available to tackle the virus infection (1). Apart from coronavirus infection, seasonal Influenza epidemics cause around 3-5 million cases of illness and up to 650,000 deaths globally (2, 3). Strategies to prevent and treat these viral infections have been limited to the development of vaccine with a relatively small arsenal of antiviral drugs. Given the emergence of large number of emerging as well as reemerging viral pathogens, which are often lethal to human, the traditional 'one bug-one drug' approach is unlikely to result in an effective therapy (4). Moreover, various subtypes of influenza virus such as Type A(H1N1) pdm2009, A(H3N2), A(H7N9) and A(H5N1) exhibited resistance to many approved antiviral drugs, such as oseltamivir and zanamivir (5-9). Thus, the development of broad-spectrum antivirals is urgently needed to control pandemic viral infections $(4,10)$. 
The broad-spectrum antivirals target some common but essential feature of viral life cycle and are beneficial over the strategies that are conventionally focused on individual viruses or specific target protein. Notably, a vast majority of the viral pathogens such as smallpox virus, flu virus, HIV, flavivirus (dengue, ebola, zika), coronavirus and arbovirus, are membraneenveloped viruses (11). To replicate inside the host cells, these classes of viruses largely rely on a common biological process namely 'membrane fusion'. The fusion between virus and host cellular or subcellular membranes is a molecular choreography between viral fusion proteins and host cell components $(12,13)$. There are underlying biophysical and biochemical features of the membrane fusion that shows marked similarity among the wide range of enveloped viruses. Thus, blocking of the classical feature of 'membrane fusion' has emerged as a 'new paradigm' for the development of broad-spectrum antivirals (4).

Lipids such as phosphatidylethanolamine (PE), diacylglycerol (DAG), oleic acid induce negative curvature at outer leaflet of membrane and promote fusion (14). Conversely, enrichment of the outer leaflet with lipids such as lysophosphatidylcholine and lysophosphoglycans, that favour positive curvature, prevent fusion of enveloped viruses (1416). Unfortunately, these fusion inhibitors (lysolipids) tend to disrupt the membranes, are often cytotoxic and rapidly metabolizable; which prevent their application as antivirals. Recently, the mechanism of actions of lysolipid helped to develop rigid amphipathic fusion inhibitors (RAFIs) (17). RAFIs were designed on the basic principle based on their ability to promote positive curvature, thus inhibiting fusion of several enveloped viruses at nanomolar concentration. However, the exact mechanism of RAFI action is unclear and possibility of geometric curvature-oriented mechanism and photosensitization induced generation of singlet oxygen were proposed (18). More importantly no clinical data or in vivo efficacy data is available with RAFIs to date (19). Therefore, the need for a cost effective broad-spectrum fusion inhibitor to tackle the viral infection in the face of an outbreak is very critical. In the present study we aim to explore how mycobacterium evades the phagosome-lysosome fusion to design an effective fusion inhibitor.

An interesting example is the case of the survival of Mycobacterium tuberculosis inside the phagosome by avoiding lysosomal degradation (20, 21). Ferrari et al. reported that Coronin 1 protein recruited at the phagosomal membrane plays a crucial role to the survival of mycobacteria inside macrophage $(22,23)$. Although, several other biochemical pathways were proposed regarding the mycobacterial evasion of phagosome-lysosome fusion (20) the role of coronin 1 to inhibit fusion cannot be undermined $(22,24,25)$. We hypothesize that structural analysis of coronin-1 coat protein will pave way to the development of a novel 
fusion inhibitor. Analysis of the 3D structure suggest that the coronin 1 have multiple tryptophan-aspartic acid domains (WD40) (26). Interestingly, it was found that the tryptophan-aspartic acid (WD) moiety is exposed outside the distorted $\beta$-meander motif (Fig. 1). Minimalistic approach suggests a lipidated WD dipeptide (Fig. 1) might possess similar activity as coronin 1 to inhibit the fusion. Therefore, in the present study we have synthesized a series of lipopeptides and ask i) what is the minimum sequence to exhibit effective inhibition of fusion? and ii) if the lipopeptide inhibits fusion, what is the mechanism of inhibition, and iii) whether the inhibition may be used to tackle the infection mediated by one of the major emerging viral pathogen of global importance, such as influenza?

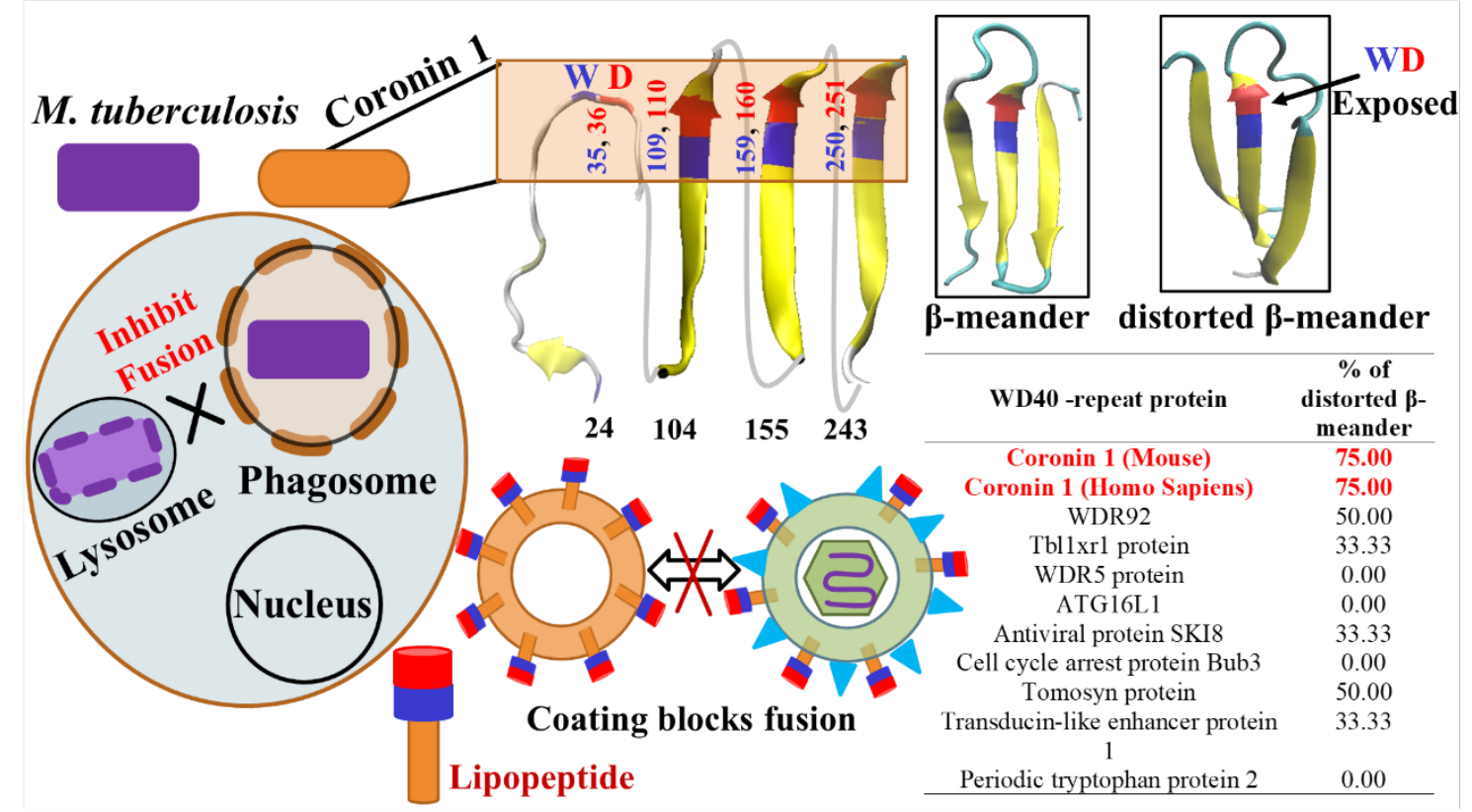

Figure 1: Phagosomal (M. tuberculosis infected) coat protein coronin 1 inhibit the fusion with lysosome. Coronin-1 structure shows multiple trp-asp sequence (WD) are exposed and mostly reside at the edge of distorted $\beta$-meander motif (blue: trp, red: asp). A lipidated dipeptide coating was hypothesized to inhibit membrane fusion between liposomes and enveloped virus. Comparison of structures of other WD40-repeat proteins suggest that coronin 1 is unique and possess highest percentage of trp-asp resides at exposed and distorted $\beta$-meander motif (see Table S1 for more details).

\section{Results and Discussion}

The N-terminus of coronin 1 composed of WD40-repeat domain. This domain is one of the abundant repeat domains and are indicative of $\beta$-propeller architecture typically comprising of seven 'blades'(27, 28). With an overall doughnut shape, the WD40 domain is among the most interacting domains in eukaryotic genomes (27-29). The analysis of the 3-dimentional 
structure of murine coronin 1 suggest that the trp-asp dipeptide sequence of the protein was placed mostly at the distorted $\beta$-meander motif (30). There are four trp-asp (WD) /WE (trpglu) sequence in murine coronin 1 and all of them (sequence 35-36; 109-110; 159-160 and 250-251) were exposed outside and not buried (Fig. 1, Fig. S2). Interestingly, 75\% of them were placed at distorted $\beta$-meander motif, where the $3^{\text {rd }}$ anti-parallel $\beta$-strand H-bonded poorly with $2^{\text {nd }} \beta$-strand and the residues (blue: trp, red: asp/glu) at the edge were more exposed (Fig. 1, Fig. S1). Now the question that rises is whether the preference of trp-asp sequence at distorted $\beta$-meander motif is unique to coronin 1 or a common feature in other WD40-repeat domain proteins! To investigate, crystal structures of eight WD40-repeat proteins were analyzed (Table S1, Fig. 1). It was found that in most cases the WD sequence was placed at a perfect $\beta$-meander motif and WD is less exposed (Fig. 1). It is worthwhile to mention that none of the eight proteins possess any fusion modulatory role (Table S1). It appears that coronin 1 structure is special and the more exposed trp-asp might have role in fusion inhibition (Fig. 1). Further, we have built the 3D model of human coronin 1 using ITASSER server (31) and noticed that 75\% of the WD/WE were placed at exposed distorted $\beta$-meander motif (Fig. S3). Apart from coronin 1, we looked into other WD40-repeat protein having membrane fusion modulatory role. The protein, tomosyn with N-terminal WD40 repeat was reported to impair the membrane bending activity of synaptotagmin-1 to inhibit SNARE complex-mediated membrane fusion (32). The model of tomosyn was built in ITASSER and it was found that $50 \%$ of trp-asp of tomosyn reside t distorted $\beta$-meander motif (Fig. S10). The structural analysis of eleven WD40-repeat proteins clearly point out that the trp-asp is more exposed in fusion inhibitory coronin 1 and tomosyn and mostly less exposed in other proteins (Fig. 1, Table S1). These findings led us to explore the effect of trp-asp (WD) and similar small dipeptides towards inhibition of fusion. Therefore, inspired by the coronin 1 structure, we have synthesized a dipeptide containing tryptophan and aspartic acid (WD) and investigated its role in membrane fusion.

Approximately $50 \mathrm{~nm}$ vesicles composed with DOPC/DOPE/Chol (55/25/20) were incubated at $\mathrm{pH} 5.5$ and the fusion was triggered by $6 \%(\mathrm{w} / \mathrm{v})$ polyethylene glycol (PEG) (33). It was noticed that $6 \%(\mathrm{w} / \mathrm{v})$ PEG has no influence on the tryptophan fluorescence intensity of the WD suggesting that PEG does not significantly alter the microenvironment of WD. The control vesicles exhibit 25\% fusion and addition of WD in membrane does not significantly change the extent of fusion (Fig. 2A). This suggests that WD peptide may not be a close mimic to coronin 1 as it cannott inhibit fusion. We recall that the coronin 1 is a coat protein, anchored at the surface and the WD region is exposed outside the membrane. We hypothesize 
that, to exert the biological activity the synthetic mimic (dipeptide) must be anchored/bound to the surface. The binding affinity of the WD peptide was evaluated by monitoring the change in the intensity of tryptophan fluorescence with an increasing concentration of membrane. It was found that the WD peptide does not bind to membrane and therefore does not influence the fusion. To accomplish a surface anchoring, we introduced lipidation at Nterminus of WD peptide. $N$-acyl tryptophan aspartic acid ( $N$-acylWD) was synthesized by acylating the $N$-terminus of tryptophan with fatty acyl chains (n=12-16, even). The binding affinity of lauroyl-WD (lau-WD), myristoyl-WD (myr-WD) and palmitoyl-WD (pal-WD) to membrane was determined by fluorescence spectroscopy (34) and listed in Table S2. It was found that all the lipo-peptide anchors to the membrane and the equilibrium dissociation constant (Kd) decreases with the increase of acyl chainlength (Table S2). After, the membrane anchoring abilitity of lipo-peptides, we examined the effect of membrane fusion. Interestingly, the lipo-peptides also inhibited fusion. The myr-WD and pal-WD was more effective than lau-WD and the inhibition nicely correlates with the membrane binding (Fig. 2B, Table S2). Since, the hydrophobic anchor plays significant role to inhibit fusion, we asked what is the role of cholesterol anchor as compared to fatty acyl anchor. The chol-WD was synthesized and the binding constant was measured. We noticed that the chol-WD bound membrane weakly as compared to myr-WD. Similarly, chol-WD couldnot inhibit the fusion effectively. We conclude that the hydrophobic anchor is imporant and myristoyl chain is the smallest anchor to exert reasonable inhibition of fusion. Therefore, further experiments were performed with myristoyl chains.Next the roles of tryptophan and aspartic acid were investigated. myr-WD dimethylester (myr-WDMe, deviod of two -COOH), myristorl tryptophan (myr-W, no aspartic acid), myristoyl aspartic acid (myr-D, no tryptophan) were synthezied and their role in fusion was investigated (Fig. 2A, 2C). The myr-WDMe couldnot block the fusion, suggesting the important role of terminal acid moiety (Fig. 2C). Now the next question that rises is what is the role of tryptophan? Both myr-D and myr-W inhibited the fusion and the extent of inhibition is slightly more with myr-W (Fig. S23). These suggest that although the myr-D have two carboxylic acid, the myr-W with one carboxylic acid is more potent, infering an important role of tryptophan. However, both the peptides were less effective than myr-WD, suggesting that the WD sequence is unique for effective fusion. MyrFD and myr-GD peptides were synthesized to evaluate the role of aromatic amino acid. myrFD inhibits more than myr-GD, suggesting than the aromatic amino acid may be a more potent inhibitor (Fig. 2D). However, comparing all the peptides it was observed myr-WD is 
the most effective inhibitor than other peptides. We conclude that the myristoyl chain, tryptophan and aspartic acid worked in tandem to exhibit effective fusion inhibition.

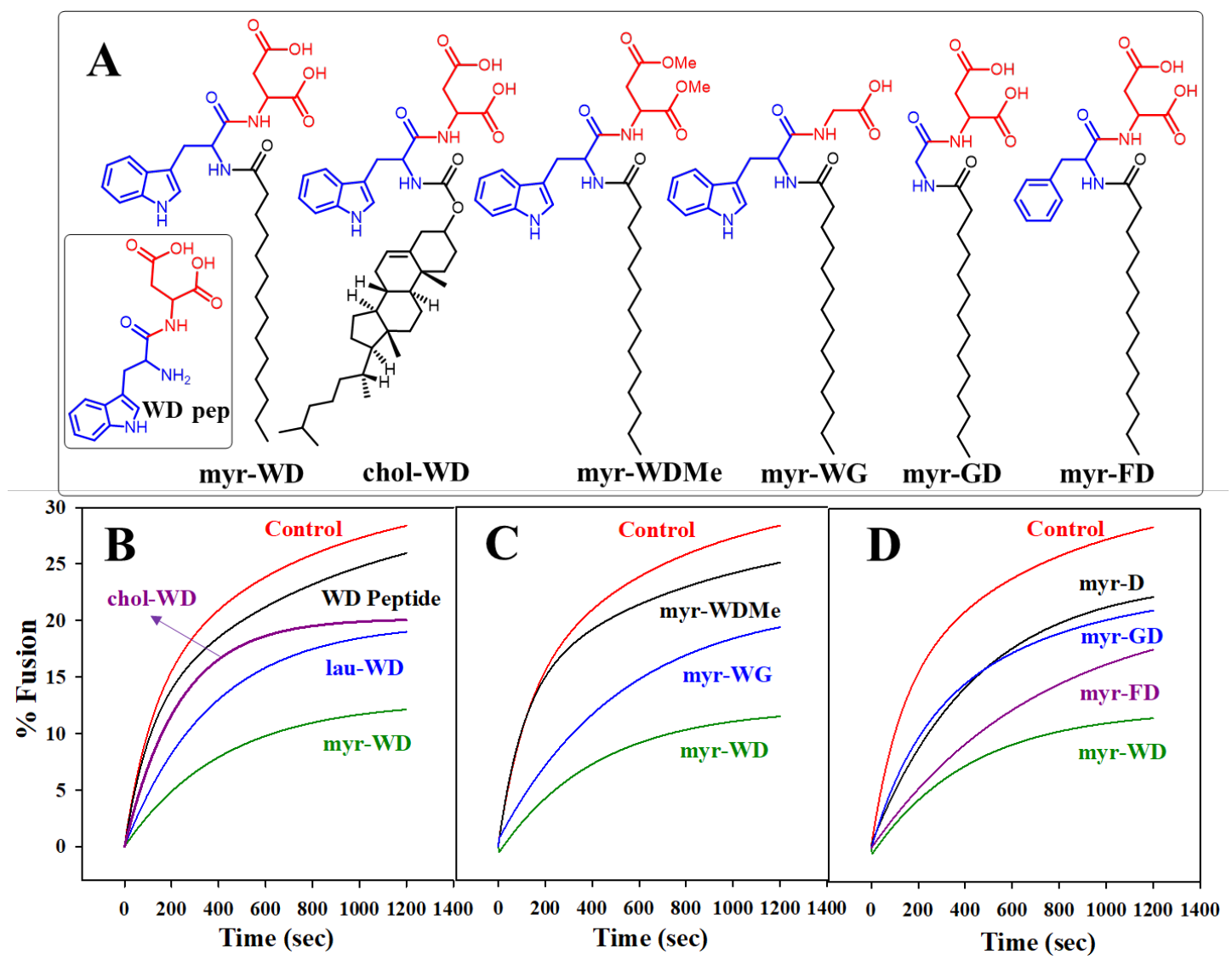

Figure 2: (A) Structures of several lipidated dipeptide explored in the present study. Effect of coronin 1 inspired lipo-peptides on the inhibition of fusion at a lipid to peptide ratio of 100:1. All the measurements were carried out in $20 \mathrm{mM}$ MES, $50 \mathrm{mM} \mathrm{NaCl}, \mathrm{pH} 5.5$ at a total lipid concentration of $200 \mu \mathrm{M}$. (B) role of lipid chain anchor, (C) role of aspartic acid and (D) role of tryptophan of lipo-dipeptide.

We recall that the WD sequence of coronin 1 is at the edge of the $\beta$-sheet and our results suggest that the specific sequence also acts as the best inhibitor of fusion. Now the question is whether the myr-WD peptide work in tandem and what is the effective lipid: peptide ratio for fusion? Concentration dependent fusion kinectics were performed and it was found that the myr-WD peptide inhibit fusion in a concentartion dependent manner (Fig. 3A). The initial rate and the extent of fusion was shown in Fig. S24 and it was observed that myr-WD effectively inhibit fusion at a peptide to lipid ratio of 1: 100. The size of the vesicles at various lipid: peptide ratio was determined to rule out effect of curvature in the fusion. The dynamic light scattering data sugest that the size of vesicles does not change significantly by the addtion of peptide at even 1:50 ratio. 
To investigate how the unique lipopeptide myr-WD inhibit fusion, extensive biophysical characterization with three distinct peptides (myr-WD, myr-WDMe and myr-GD) were performed. It was noticed earlier that myr-WD inihibit the fusion, myr-WDMe has no role in fusion and myr-GD slightly inhibit the fusion. The membrane order at the interfacial region was measured at various peptide to lipid ratio by monitoring the steady-state fluorescence anisotropy of a fluorophore, TMA-DPH. The TMA-DPH anisotropy increased with the increase of myr-WD peptide in membrane. myr-GD slightly increased the anisotropy, whereas addition of myr-WDMe in membrane did not change the anisotropy (Fig. 3B). The increase in TMA-DPH anisotropy by the most effective fusion inhibitory peptide myr-WD indicates that the peptide effectively order the membrane interface (35). Since ordering of membrane interface inhibits the lipid protrusion required for hemifusion, we suggest that myr-WD inhibit the fusion by ordering the membrane interface. Next, the water penetration to the bilayer was determined at various peptide to lipid ratio by measuring the lifetime of TMA-DPH. The average lifetime increased with the addition of two fusion inhibitory peptide, myr-GD and myr-WD in membrane, whereas the average lifetime did not increase with the addition of myr-WDMe (Fig. 3C). The increase in average lifetime suggests that water penetration into the membrane decreases with the addition of fusion inhibitory peptides (36, 37). Membrane fusion is generally believed to proceed via lipid-water mixture to form hemisfusion intermediate. Therefore, reduction of water penetration in membrane by the myrWD peptide likely interfere with the lipid protrusion required for hemifusion $(36,38)$. Next the zeta potential was measured as the surface potential was shown earlier to modulate the fusion (39). It was observed that while myr-WDMe does not change the zeta potential with increasing the peptide concentration, myr-WD increases the zeta potential. The other peptide, myr-GD also increases the zeta potential but not at the same extent as myr-WD. It is interesting to note that although both myr-GD and myr-WD have two -COOH group with identical charged headgroup at interface, the extent of reduction of zeta potential with myrWD is almost twice as compared to myr-GD (Fig. 3D). The more negative zeta potential of myr-WD may be responsible for the inhibition as the negative surface potential was reported to inhibit the fusion (39). All these experiments suggest that myr-WD is unique peptide sequnce and it effectively inhibit the fusion by the increase in the interfacial order, zeta potential and decrease in water penetration. It may be suggested that the unique nature of WD sequence was chosen in coronin 1 to inhibit phagosome-lysozome fusion. 

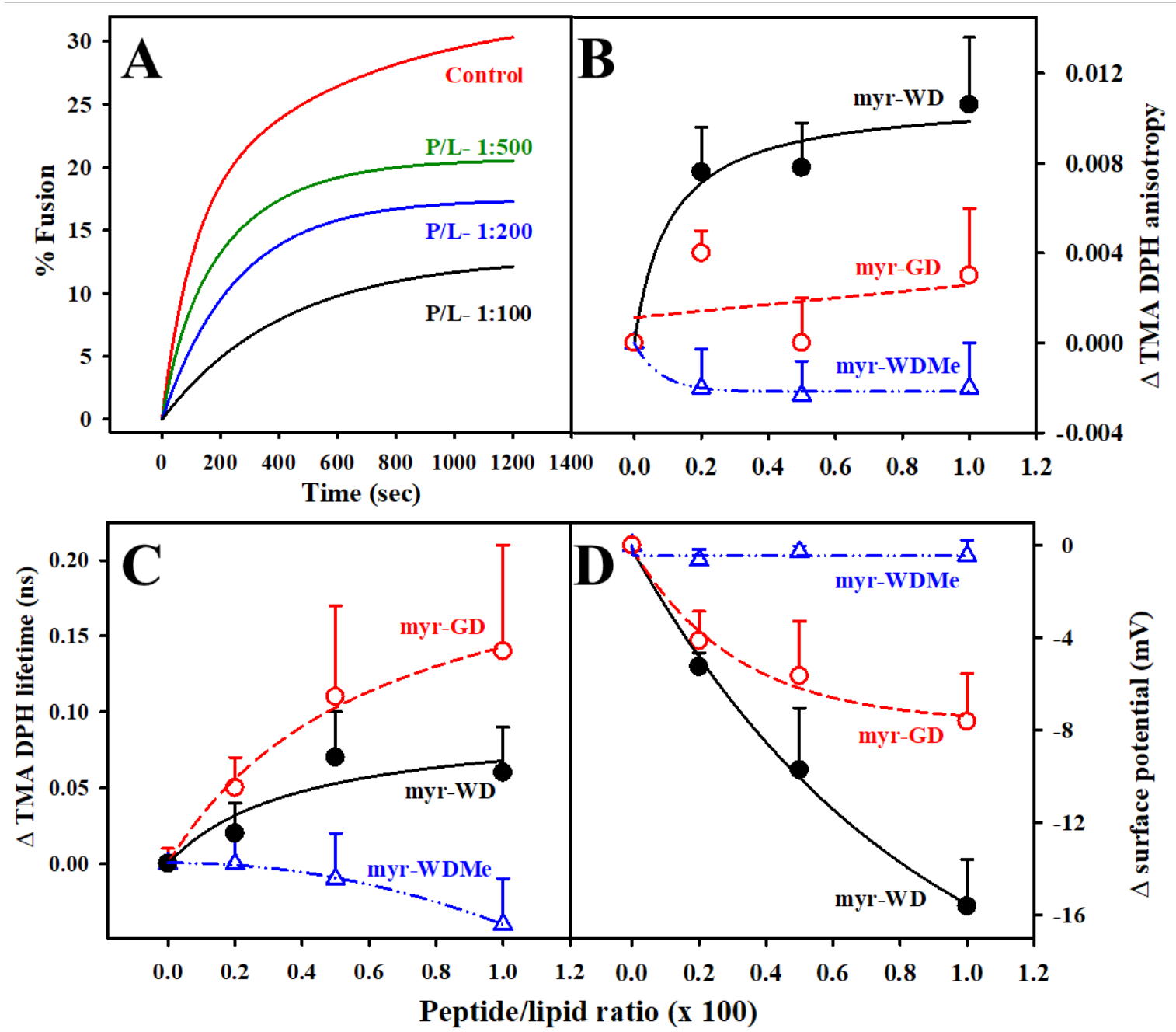

Figure 3: Mechanistic studies on the inhibition of fusion. (A) myr-WD peptide inhibit the fusion in a concentration dependent manner, (B) change in the fluorescence anisotropy of TMA-DPH in DOPC/DOPE/Chol (55/25/20) membranes with increasing concentration of myr-WD peptide (black, •), myr-GD peptide (red, o) and myr-WDMe peptide (blue, $\Delta$ ), (C) change in the average fluorescence lifetime of TMA-DPH, (D) change in the zeta potential with increasing concentration of the above peptides. The lines were drawn to guide the eye.

Since the myr-WD peptide effectively inhibits fusion in vitro, it thus might inhibit viral infection by inhibiting membrane fusion. The life-cycle of enveloped viruses heavily dependent on membrane fusion and the potential of myr-WD and myr-GD peptide against H1N1 virus infection was tested as a model to address the broader goal of our present study. Briefly, the antiviral activity of myr-WD peptide was tested in MDCK (Madin-Darby Canine Kidney) cells against avian influenza type A/PR/8/34 (H1N1) virus challenge by standard plaque assay as well as MTT based cell survivability assay (Fig. 4). It was observed that 200 $\mu \mathrm{M}$ myr-WD exhibited a low cytotoxicity against MDCK cell $\left(\mathrm{CC}_{50}\right)$. Next, the infection of 
MDCK cells $\left(0.05 \times 10^{6}\right.$ cells per well) was initiated with $100 \mathrm{MOI}$ of virus for $2 \mathrm{hr}$ at $37{ }^{0} \mathrm{C}$ under $5 \% \mathrm{CO}_{2}$ pressure in a humidified chamber. Significant reduction in viral plaque formation was observed with the addition of myr-WD peptide (Fig. 4A). MTT based cell survivability assay also supports that the designed lipo-peptide protects the cells from viral challenge at low concentration ( $\sim 3 \mu \mathrm{M})$ (Fig. 4B). The corresponding cell cytopathic effect (CPE) characterized by rounding up and detachment of cells, was observed by inverted light microscope. The reduction of CPE in myr-WD treated cells suggested that the fusion inhibitory myr-WD provided protection against influenza viral infection. The cells treated with the peptide were observed to retain their cellular morphology as compared to cells that did not receive myr-WD treatment (Fig 4C). Altogether, the data presented herein demonstrate the potential of myr-WD peptide as a new class of bioinspired protection agent against influenza virus.
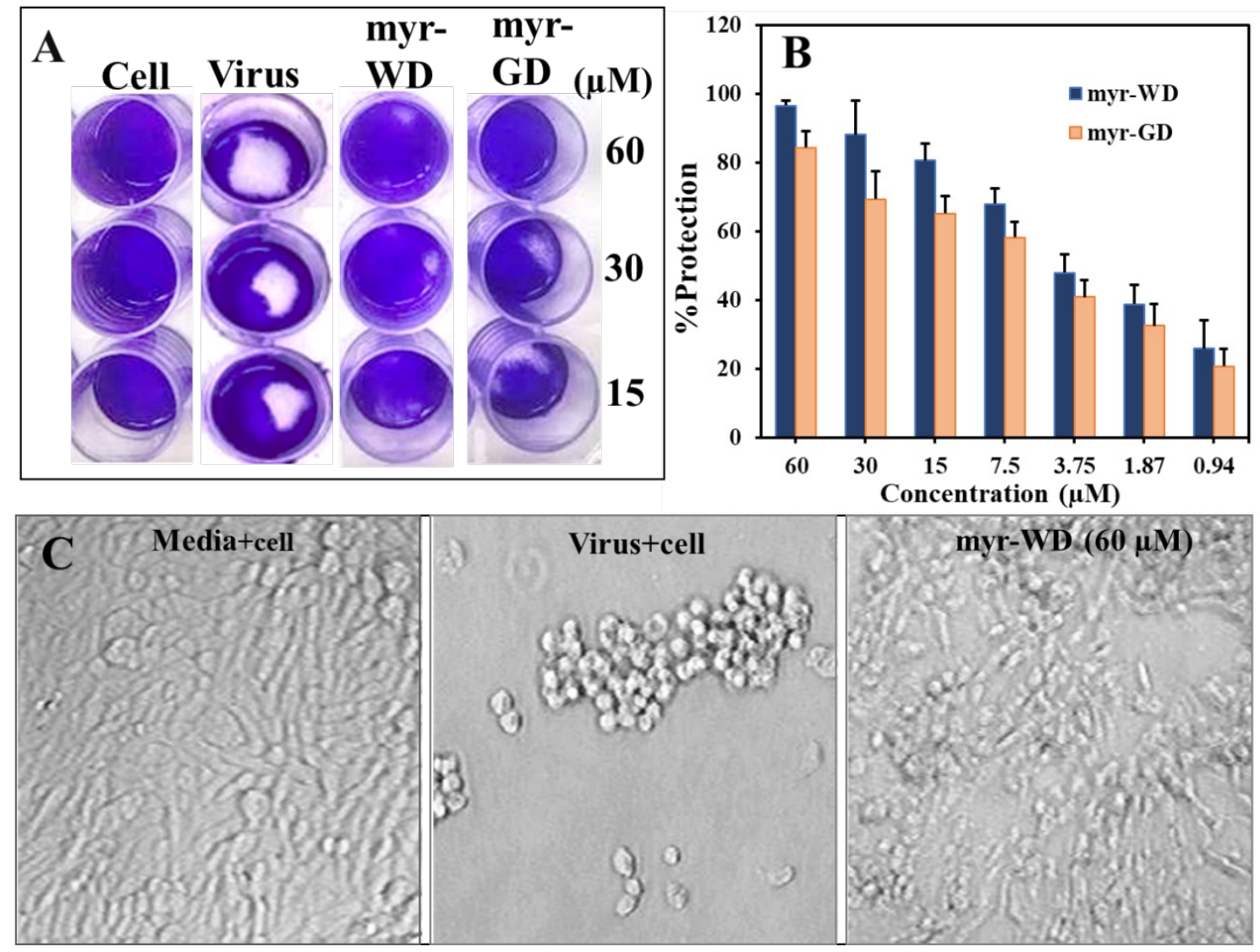

Figure 4: myr-WD protect the influenza-A virus infection. (A) myr-WD reduce viral plaque formation in avian influenza type A/PR/8/34 (H1N1) virus infected MDCK cells in a dosedependent manner. (B) myr-WD provide protection against influenza viral infection in H1N1virus-infected MDCK cells (MOI = 100) evaluated by $(\mathrm{B})$ MTT assay and $(\mathrm{C})$ image of the cells viewed under inverted light microscope (Nikon, Japan) at 20X magnification. 
Infectious diseases of viral origin routinely affect the human civilization. The most effective ways to treat the viral infection is to develop vaccines. However, the emergence of new viruses and their potential mutations obstruct the effectivity of vaccines. For example, the recent coronavirus infection already killed $~ 251000$ people worldwide and no vaccine will likely be in the market until next year. This suggests that the development of a broadspectrum anti-viral is essential to tackle viral infections $(4,10)$. The broad-spectrum antivirals are expected to be superior than traditional 'one drug one bug' approach as the drug development process always has some lag period. To develop broad-spectrum antivirals, scientists target some essential biological features which are common in many viruses. One such important process is the viral entry into the host cell via fusion between the viral membrane and host cell. While fusion is not a spontaneous process and viruses have sophisticated protein machinery to pursue the energetically uphill task $(12,33)$. Drug target to the fusion machinery likely be specific to a particular virus and may not be useful to target for a broad-spectrum antiviral agent. Instead the membrane active compounds that passively interferes the membrane fusion likely be new class of molecules to inhibit fusion and may be used as broad-spectrum anti-viral agent (17). To inhibit the fusion and search for a membrane active compound, we hypothesized that the coronin 1 survival strategy may be translated to an effective fusion inhibitor as coronin 1 of mycobacterium helps to evade the fusion between phagosome and lysosome (22).. The 3D structure of various WD-40 repeat protein including coronin 1 suggest that the WD is placed mostly at the edge of $\beta$-sheet, placed in a distorted $\beta$ meander and WD more exposed to outside. Several WD lipopeptides were synthesized and their fusion inhibition potential were evaluated. It was found that the myristoyl chain helps to anchor the peptide in membrane and the WD sequence is unique to inhibit the fusion. The myr-WD inhibit the fusion by increasing the interfacial order, decrease in water penetration and increase in surface potential. Interestingly, myr-WD also effective to tackle the H1N1 infection at relatively low concentration $(\sim 3 \mu \mathrm{M})(40)$. The membrane active nature of the peptide likely reflects the micromolecular concentration required to combat H1N1 infection. We suggest that the mycobacterium inspired approach is completely new and will likely pave way to develop broad-spectrum antiviral to tackle several deadly viruses such as influenza, coronavirus, ebola etc.

\section{Conclusions}

The emergence of highly pathogenic viruses questions the unparallel superiority of vaccines as the process of vaccine development is associated with substantial amount of lag period with relatively high cost. To this end, a fusion inhibitor may emerge as an effective broad- 
spectrum antiviral as membrane fusion is important to the life cycle of many enveloped viruses. Here we utilized the survival of mycobacterium inside the phagosome to develop a new class of fusion inhibitor. Mycobacterium coats the phagosome with coronin 1 to inhibit fusion with lysosome. Structural analysis of coronin 1 and other WD40-repeat proteins suggests that the microenvironment of trp-asp (WD) sequence in fusion inhibitory protein is unique. This has inspired us to explore the role of several lipo-dipeptides and finally identify an effective small-molecule fusion inhibitor, myr-WD that recapitulates the likely feature of coronin 1 . We demonstrated that myr-WD increases interfacial order and decreases water penetration, surface potential to inhibit membrane fusion. The mycobacterium inspired simple lipo-dipeptide was shown to protect cells from lethal type 1 influenza virus (H1N1) viral challenge as a 'broad spectrum' antiviral agent.

\section{Acknowledgements}

PKT acknowledges support from DST-Inspire (IFA13-CH-120, 2014) and the Science and Engineering Research Board (SERB) Early Career Award (ECR/2016/001935). AS thanks UGC and AL thanks IISER Kolkata for fellowship support. PKT and AIM thanks IISER Kolkata for infrastructure and financial support.

\section{References}

1. R. Lane, Sarah Gilbert: carving a path towards a COVID-19 vaccine. Lancet 395, 1247 (2020).

2. M. J. P. Van Dongen, et al., A small-molecule fusion inhibitor of influenza virus is orally active in mice. Science. 363 (2019).

3. , World Health Organization (WHO), WHO influenza (seasonal) fact sheet (2018). https://www.who.int/news-room/fact-sheets/detail/influenza-(seasonal).

4. F. Vigant, N. C. Santos, B. Lee, Broad-spectrum antivirals against viral fusion. Nat. Rev. Microbiol. 13, 426-437 (2015).

5. S. S. Liu, et al., Susceptibility of influenza A(H1N1)/pdm2009, seasonal A(H3N2) and B viruses to Oseltamivir in Guangdong, China between 2009 and 2014. Sci. Rep. 7, 111 (2017).

6. R. Hai, et al., Influenza A(H7N9) virus gains neuraminidase inhibitor resistance without loss of in vivo virulence or transmissibility. Nat. Commun. 4, 2854 (2013).

7. N. X. Wang, J. J. Zheng, Computational studies of H5N1 influenza virus resistance to oseltamivir. Protein Sci. 18, 707-715 (2009).

8. M. D. De Jong, et al., Oseltamivir resistance during treatment of influenza A (H5N1) infection. N. Engl. J. Med. 353, 2667-2672 (2005). 
9. H. Marjuki, et al., Neuraminidase Mutations Conferring Resistance to Oseltamivir in Influenza A(H7N9) Viruses. J. Virol. 89, 5419-5426 (2015).

10. V. Boldescu, M. A. M. Behnam, N. Vasilakis, C. D. Klein, Broad-spectrum agents for flaviviral infections: Dengue, Zika and beyond. Nat. Rev. Drug Discov. 16, 565-586 (2017).

11. F. A. Rey, S. M. Lok, Common Features of Enveloped Viruses and Implications for Immunogen Design for Next-Generation Vaccines. Cell 172, 1319-1334 (2018).

12. R. K. Plemper, Cell entry of enveloped viruses. Curr. Opin. Virol. 1, 92-100 (2011).

13. F. L. Cosset, D. Lavillette, Cell Entry of Enveloped Viruses. Adv. Genet. 73, 121-183 (2011).

14. R. M. Epand, R. F. Epand, N. Ahmed, R. Chen, Promotion of hexagonal phase formation and lipid mixing by fatty acids with varying degrees of unsaturation. Chem. Phys. Lipids 57, 75-80 (1991).

15. M. J. Hope, P. R. Cullis, The role of nonbilayer lipid structures in the fusion of human erythrocytes induced by lipid fusogens. BBA - Biomembr. 640, 82-90 (1981).

16. M. M. Kozlov, S. L. Leikin, L. V. Chernomordik, V. S. Markin, Y. A. Chizmadzhev, Stalk mechanism of vesicle fusion - Intermixing of aqueous contents. Eur. Biophys. $J$. 17, 121-129 (1989).

17. M. R. St. Vincent, et al., Rigid amphipathic fusion inhibitors, small molecule antiviral compounds against enveloped viruses. Proc. Natl. Acad. Sci. U. S. A. 107, 1733917344 (2010).

18. F. Vigant, et al., The Rigid Amphipathic Fusion Inhibitor dUY11 Acts through Photosensitization of Viruses. J. Virol. 88, 1849-1853 (2014).

19. J. A. Jackman, P. Y. Shi, N. J. Cho, Targeting the Achilles Heel of Mosquito-Borne Viruses for Antiviral Therapy. ACS Infect. Dis. 5, 4-8 (2019).

20. M. Mori, R. Mode, J. Pieters, From phagocytes to immune defense: Roles for coronin proteins in dictyostelium and mammalian immunity. Front. Cell. Infect. Microbiol. 8 (2018).

21. J. Pieters, Mycobacterium tuberculosis and the Macrophage: Maintaining a Balance. Cell Host Microbe 3, 399-407 (2008).

22. G. Ferrari, H. Langen, M. Naito, J. Pieters, A coat protein on phagosomes involved in the intracellular survival of mycobacteria. Cell 97, 435-447 (1999).

23. R. Jayachandran, et al., Survival of Mycobacteria in Macrophages Is Mediated by Coronin 1-Dependent Activation of Calcineurin. Cell 130, 37-50 (2007). 
24. L. Nguyen, J. Pieters, The Trojan horse: Survival tactics of pathogenic mycobacteria in macrophages. Trends Cell Biol. 15, 269-276 (2005).

25. G. P. Pattnaik, H. Chakraborty, Coronin 1 derived tryptophan-aspartic acid containing peptides inhibit membrane fusion. Chem. Phys. Lipids 217, 35-42 (2018).

26. B. A. Appleton, P. Wu, C. Wiesmann, The crystal structure of murine coronin-1: A regulator of actin cytoskeletal dynamics in lymphocytes. Structure 14, 87-96 (2006).

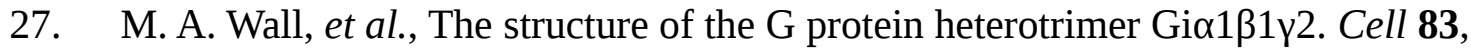
1047-1058 (1995).

28. C. Xu, J. Min, Structure and function of WD40 domain proteins. Protein Cell 2, 202214 (2011).

29. M. Schapira, M. Tyers, M. Torrent, C. H. Arrowsmith, WD40 repeat domain proteins: A novel target class? Nat. Rev. Drug Discov. 16, 773-786 (2017).

30. B. A. Appleton, P. Wu, C. Wiesmann, The crystal structure of murine coronin-1: A regulator of actin cytoskeletal dynamics in lymphocytes. Structure 14, 87-96 (2006).

31. Y. Zhang, I-TASSER server for protein 3D structure prediction. BMC Bioinformatics 9 (2008).

32. Y. Yamamoto, et al., Tomosyn inhibits synaptotagmin-1-mediated step of Ca2+dependent neurotransmitter release through its N-terminal WD40 repeats. J. Biol. Chem. 285, 40943-40955 (2010).

33. J. Lee, B. R. Lentz, Secretory and viral fusion may share mechanistic events with fusion between curved lipid bilayers. Proc. Natl. Acad. Sci. U. S. A. 95, 9274-9279 (1998).

34. V. Koppaka, B. R. Lentz, Binding of bovine factor Va to phosphatidylcholine membranes. Biophys. J. 70, 2930-2937 (1996).

35. M. E. Haque, et al., Hemagglutinin fusion peptide mutants in model membranes: Structural properties, membrane physical properties, and PEG-mediated fusion. Biophys. J. 101, 1095-1104 (2011).

36. H. Chakraborty, P. K. Tarafdar, D. G. Klapper, B. R. Lentz, Wild-type and mutant hemagglutinin fusion peptides alter bilayer structure as well as kinetics and activation thermodynamics of stalk and pore formation differently: Mechanistic implications. Biophys. J. 105, 2495-2506 (2013).

37. A. P. Demchenko, Y. Mély, G. Duportail, A. S. Klymchenko, Monitoring biophysical properties of lipid membranes by environment-sensitive fluorescent probes. Biophys. J. 96, 3461-3470 (2009). 
38. P. M. Kasson, E. Lindahl, V. S. Pande, Water ordering at membrane interfaces controls fusion dynamics. J. Am. Chem. Soc. 133, 3812-3815 (2011).

39. P. K. Tarafdar, H. Chakraborty, S. M. Dennison, B. R. Lentz, Phosphatidylserine inhibits and calcium promotes model membrane fusion. Biophys. J. 103, 1880-1889 (2012).

40. F. Vigant, et al., A Mechanistic Paradigm for Broad-Spectrum Antivirals that Target Virus-Cell Fusion. PLoS Pathog. 9, e1003297 (2013). 
